\title{
Ultrasound guidance showing real-time local anesthetic extravasation during injection of two lateral popliteal sciatic nerve blocks
}

\author{
Brian Osman, Andres Missair, Robyn S. Weisman, Catalina Castillo-Pedraza, Ralf E. Gebhard \\ Department of Anesthesiology, Miller School of Medicine, University of Miami, Miami, FL, USA \\ Correspondence: Andres Missair. Address: 193 N. Shore Dr. \#602, Miami Beach, FL 33141, USA. Telephone: \\ 01-305-310-2872. Email: amissair@med.miami.edu.
}

Received: May 2, 2013

DOI : $10.5430 / j b g c . v 3 n 4 p 70$
Accepted: June 3, $2013 \quad$ Online Published: July 19, 2013

URL: http://dx.doi.org/10.5430/jbgc.v3n4p70

\section{Abstract}

Objective: The purpose of this case report is to describe an observation of local anesthetic extravasation while performing single-shot lateral sciatic popliteal blocks on two patients using ultrasound guided imaging. This under-reported phenomenon may have clinical implications and warrants further examination of conventional practice techniques.

Case report: We performed lateral sciatic popliteal blocks under ultrasound guidance for post-operative pain in two patients having surgery in the left lower extremity. Both blocks involved sequential, real-time scans during the injection beneath the complex fascial sheath of the sciatic nerve. Observations included extrafascial extravasation of local anesthetic away from the nerve, up the needle path, and also concentrically outside the nerve sheath. Both blocks had $100 \%$ sensory block but slower clinical onset than expected.

Conclusions: Ultrasound imaging is an evolving technology gaining popularity for performing peripheral nerve blocks. The incidence and volume of local anesthetic extravasation with single shot nerve blocks are unknown. This phenomenon may be common but frequently undetected given the limited resolution and the two-dimensional nature of current ultrasound imaging technology. The clinical consequences of local anesthetic injection into tissues outside of the nerve sheath are unknown, however our observations suggest speed of onset and quality of blocks may be affected. Further investigation evaluating the extravasation of local anesthetics during ultrasound blockade is needed to re-evaluate injection speeds and the use of conventional volumes for injection.

\section{Key words}

Ultrasound guidance, Nerve block, Regional anesthesia

\section{Introduction}

Ultrasound guidance for the placement of peripheral nerve blocks has drawn interest into the possibility of increasing the safety, time, and efficacy of nerve blocks ${ }^{[1,2]}$. It is a relatively new application to the field of regional anesthesia that has introduced a great amount of anatomic detail for block techniques, but even reported success rates of up to 99\% can still show variations with the time to clinical onset and duration of the block ${ }^{[1]}$. Mean block onset, defined as motor paralysis 
and sensory anesthesia, for the lateral popliteal sciatic nerve block ranges from 15 minutes to 67 minutes ${ }^{[1-3]}$. While mean block duration for $0.5 \%$ Ropivacaine has been reported between 12 and 18 hours. One would expect that direct visualization of the nerve, needle tip, and local anesthetic deposition would contribute to a more uniform clinical presentation. The 2-D nature of ultrasound, however, limits the operator's ability to assess the complete distribution of local anesthetic during the injection. Given the clinical variability often seen, despite the use of ultrasound, there is no definitive method to predict the complete distribution of the local anesthetic when injected ${ }^{[3]}$. In this case report, we report two cases of local anesthetic extravasation observed in real-time, while performing two lateral popliteal sciatic nerve blocks. Both demonstrated $100 \%$ sensory block with incomplete motor paralysis and delayed onset. Although this is only an observation of two cases, attention should be brought to the possible clinical implications of this under-reported phenomenon.

\section{Case report}

This case report complies with section 20.1 of the University of Miami Miller School of Medicine IRB policy and procedures for publications that do not require prior IRB approval. Two male patients, ages 47 and 51, scheduled for surgery of the left lower extremity (long flexor tendon release and neuroma excision, respectively), received postoperative left lateral sciatic popliteal blocks under ultrasound guidance for postoperative pain. Informed consent was obtained from the two patients for a peripheral nerve block prior to transfer to the operating room. The blocks were performed postoperatively in the post anesthesia care unit with the patients in the supine position on supplemental oxygen via facemask and with the standard ASA monitors in place. Patients were awake and did not receive sedation. The patients were in the supine position with a pillow placed beneath the ankle for leg elevation. A BK Medical Flex Focus 400 Anesthesia ultrasound machine with a 6 to 18-MHz High Frequency Linear Transducer 8870 (BK Medical, Peabody MA) was set to obtain real-time images at $15 \mathrm{MHz}$. After sterile prep and drape, the transducer probe was placed in the popliteal fossa. The probe was positioned to obtain a transverse view of the sciatic nerve immediately proximal to the bifurcation into the tibial and peroneal branches. The nerve was visualized as hyperechoic and well-circumscribed in both patients. The point of injection was the groove created between the adjacent common peroneal and tibial branches, immediately proximal to bifurcation.

After subcutaneous infiltration with 1\% Lidocaine, a 100mm 21 gauge insulated short bevel stimulating needle (Stimuplex A; B Braun, Bethlehem, PA) was used to make a single skin puncture at the lateral aspect of the thigh, approximately $5-10 \mathrm{~cm}$ cephalad to the top of the patella, with an in-plane visualization of the needle. The needle was advanced in-plane through the biceps femoris muscle and epimysium to reach the sciatic groove. With a clear image of the needle tip and shaft, the needle was then advanced beneath the complex fascia of the sciatic nerve bifurcation, at either the 6 o'clock or 12 o'clock positions where a small separation exists between the Common Peroneal and Posterior Tibial nerve branches. A volume of $30 \mathrm{~mL}$ of $0.5 \%$ Ropivacaine was injected at a rate of $10 \mathrm{~mL} / \mathrm{min}$ without resistance. The needle tip was not redirected and a single needle pass was performed in both cases. Perineural spread was confirmed on ultrasound in real time.

The first patient's injection demonstrated local anesthetic extravasation away from the nerve and along the path of the needle (see Figure 1). Expanding pockets of local anesthetic outside of the intended target could be appreciated under the continuous real-time ultrasound scan. The injection with the second patient also showed local anesthetic extravasation away from the nerve, along the needle path, and also outside, immediately adjacent to the complex fascial sheath of the sciatic nerve (see Figure 2, 3, and 4). No resistance was appreciated with either injection and neither patient reported any pain or paresthesias. Neural swelling was not appreciated in either case. Both patients demonstrated complete sensory block at 30 minutes with incomplete motor paralysis. Block duration was 10 hours and 13 hours, respectively. 

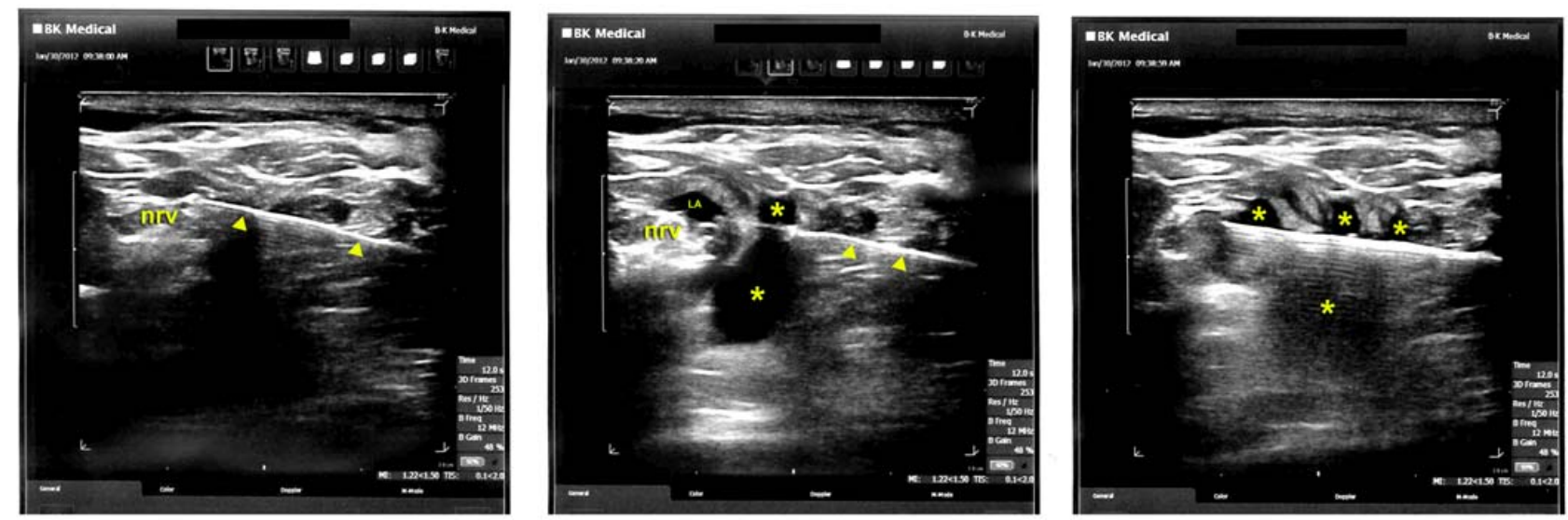

Figure 1. Sequential ultrasound transverse axis scans of the sciatic nerve (at the popliteal fossa) showing real-time local anesthetic extravasation (*) away from the nerve (nrv) and along the needle shaft / path (triangles.)
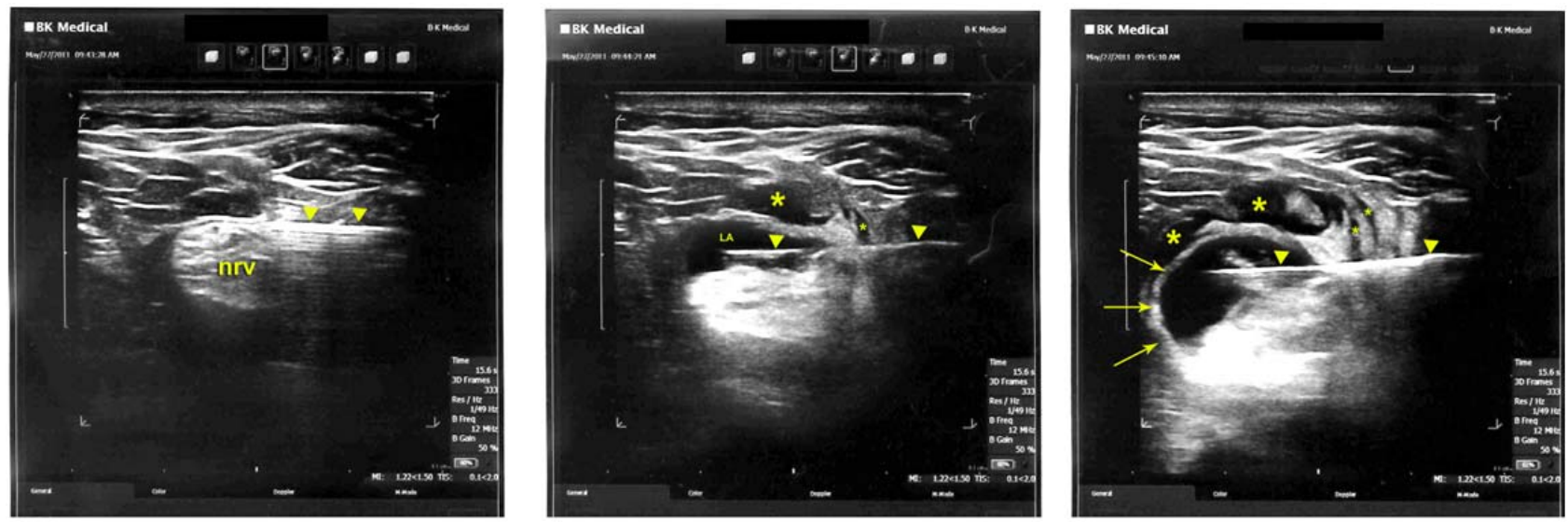

Figure 2. Sequential ultrasound transverse axis scans of the sciatic nerve (at the popliteal fossa) showing real-time local anesthetic extravasation (*) away from the nerve (nrv) and along the needle shaft/path (triangles). Extrafascial extravasation $\left(^{*}\right)$ is also seen adjacent to the complex fascial sheath of the nerve (arrows).

Figure 3. Post-block 3-D ultrasound scan along the longitudinal axis of the sciatic nerve (proximal to the popliteal fossa) showing real-time local anesthetic extravasation (white arrows) away from the nerve (SCI NRV black arrows). The extravasated local anesthetic presents in the form of discreet pockets.

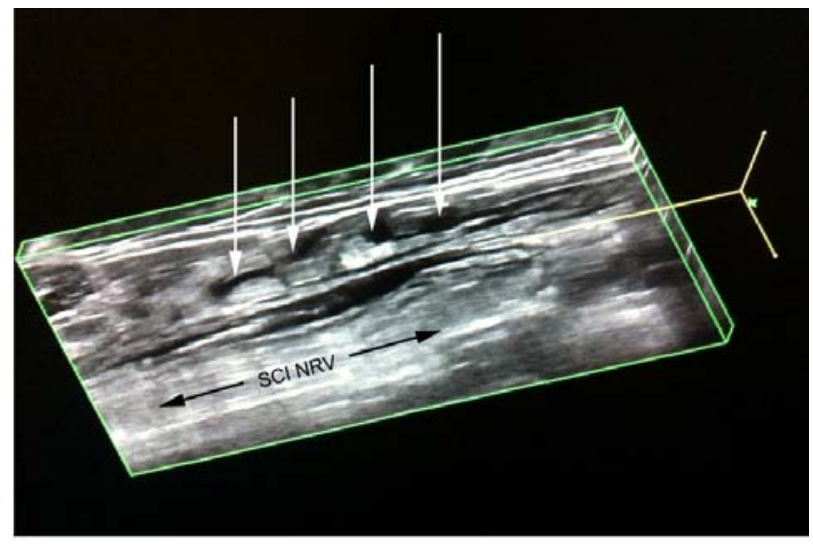


Figure 4. Post-block 3-D ultrasound scan along the longitudinal axis of the sciatic nerve (proximal to the popliteal fossa) showing real-time local anesthetic extravasation at the site of injection. The extravasated local anesthetic (white arrow) presents in the form of a longitudinal pocket parallel to the nerve (SCI NRV - black arrows). The local anesthetic in contact with the nerve, beneath the fascial sheath is identified by $(*)$.

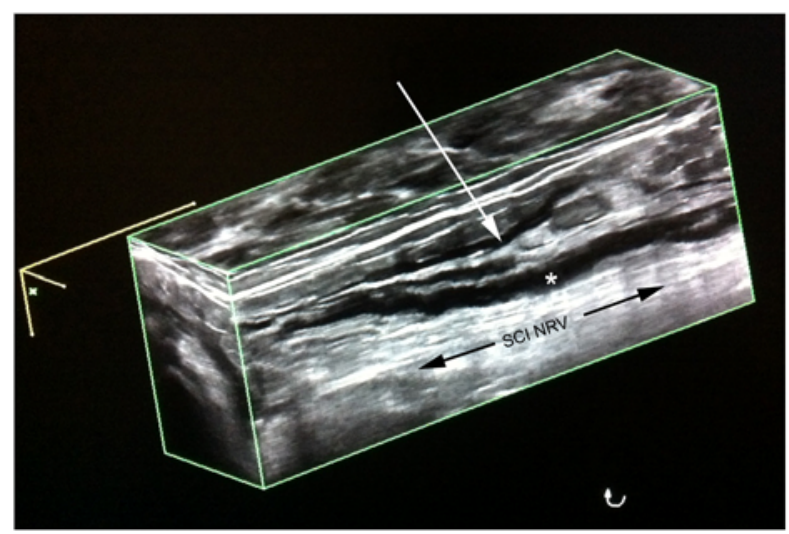

\section{Discussion}

The use of ultrasound for peripheral nerve blocks has rapidly gained popularity, but it is a still-evolving technology. Recent studies are demonstrating that the speed and success of sciatic nerve blocks are improved by the use of ultrasound but, in spite of this, there is few data that attempt to describe the ideal morphology and pattern of local anesthetic spread during injections as observed under ultrasound imaging ${ }^{[2,4,5]}$. Whereas the evidence has shown the importance of perineural circumferential spread, and the consequences of intraneural injection, the real-time, dynamic distribution of local anesthetic is relatively undefined and still debated ${ }^{[3,6]}$. Part of the dilemma is appreciating a three dimensional phenomenon using a two dimensional imaging technique. Although there are reports of using three-dimensional ultrasound to improve visualization of the anatomy with these types of peripheral nerve blocks, it still has not reached mainstream application in clinical practice, nor has there been much focus on the dynamics of the local anesthetic during injection ${ }^{[7]}$.

Managing local anesthetic extravasation around continuous infusion catheters has gained some attention but not much data is available to investigate the possibility of local anesthetic leakage while performing single-shot injections ${ }^{[8]}$. The extravasation of local anesthetic observed while performing the two popliteal sciatic nerve blocks mentioned above poses a couple of interesting questions. First, what is the incidence and how often is it overlooked? Due to the two-dimensional nature of the image and limited resolution of ultrasound technology, this phenomenon can be missed, especially if it flows up the needle path as in Figure 1 or if the image is obscured by other surrounding hypoechoic structures such as subcutaneous fat. One could argue that this phenomenon could be explained by high pressure or high injection speeds. We believe that this is unlikely, since the blocks showed extravasations in spite of the slow and controlled $10 \mathrm{~mL} / \mathrm{min}$ injections in the absence of perceptible resistance.

Although the expanding hypoechoic pockets observed from the real-time continuous scans offered compelling evidence that local anesthetic was filling spaces away from the nerve, outside the complex fascial sheath of the nerve (see Figure 2, 3 , and 4), and along the needle path, the blocks were $100 \%$ effective albeit with delayed onset. This begs the question if using conventional volumes of local anesthetic is really necessary. If the majority of the local anesthetic is pushed into spaces away from the nerve, does it contribute effectively to the block? Studies have suggested that much of the volume injected may diffuse into surrounding soft tissues or undergo vascular uptake and therefore not contribute to anesthesia ${ }^{[9]}$. Cadaveric studies by Andersen et al., specifically mention local anesthetic extravasation during their experiment involving dye injection beneath the sciatic paraneural sheath ${ }^{[10]}$. Recent work is demonstrating that much smaller amounts of local anesthetic can provide satisfactory blocks ${ }^{[5,11]}$. This evidence in conjunction with our own observations suggest that it may be possible to use a substantially lower volume of local anesthetic and achieve a successful clinical block. 
Another important consideration regarding the slower onset times for both blocks, is that the direct visualization of concentric perineural spread observed on ultrasound does not guarantee that the local anesthetic is actually in contact with the nerve, as evidenced by Figure 2. Some have suggested that ultrasound imaging does not have the ability to differentiate the epineurium from the complex fascial layer encompassing the sciatic nerve ${ }^{[4]}$. Others suggest that these two layers are the same ${ }^{[12]}$. In conclusion, this debate suggests that ultrasound technology has limitations in detecting exactly where local anesthetic is being injected in relation to tissue planes that currently require histological analysis to clearly define ${ }^{[10]}$.

The incidence and volume of local anesthetic extravasation is unknown during ultrasound-guided blockade and this area warrants further research. Future results may impact conventional local anesthetic volumes for injection, injection speeds, and elucidate the clinical consequences of local anesthetic being injected into tissues not in contact with the actual nerve.

\section{References}

[1] Sinha A, Chan VW. Ultrasound imaging for popliteal sciatic nerve block. Reg Anesth Pain Med. 2004; 29: 130-134. PMid:15029549

[2] Perlas A, Brull R, Chan VW, McCartney CJ, Nuica A, Abbas S. Ultrasound guidance improves the success of sciatic nerve block at the popliteal fossa. Reg Anesth Pain Med. 2008; 33: 259-265. PMid:18433678

[3] Morau D, Levy F, Bringuier S, et al. Ultrasound-guided evaluation of the localanesthetic parameters required for a rapid surgical popliteal sciatic nerve block. Reg Anesth Pain Med. 2010; 35: 559-564. PMid:20975475 http://dx.doi.org/10.1097/AAP.0b013e3181fa6b60

[4] Sites BD, Neal JM, Chan V. Ultrasound in regional anesthesia: Where should the "focus” be set? RegAnesth Pain Med. 2009; 34: 531-533. PMid:19901765 http://dx.doi.org/10.1097/AAP.0b013e3181c0f025

[5] Sinha SK, Abrams JH, Barnett JT, et al. Decreasing the local anesthetic volume from20 to $10 \mathrm{~mL}$ for ultrasound-guided interscalene block at the cricoids level does notreduce the incidence of hemidiaphragmatic paresis. Reg Anesth Pain Med. 2011; 36: 17-20. PMid:21751435 http://dx.doi.org/10.1097/AAP.0b013e3182030648

[6] Bigeleisen PE. Nerve puncture and apparent intaneural injection during ultrasound-guided axillary block does not invariably result in neurologic injury. Anesthesiology. 2006; 105: 779-783. PMid:17006077 http://dx.doi.org/10.1097/00000542-200610000-00024

[7] Feinglass NG, Clendenen SR, Torp KD, Wang RD, Castello R, Greengrass RA. Real-time three-dimensional ultrasound for continuous popliteal blockade: A case reportand image description. Anesth Analg. 2007; 105: 272-274. PMid:17578987 http://dx.doi.org/10.1213/01.ane.0000265439.02497.a7

[8] Gurnaney H, Kraemer FW, Ganesh A. Dermabond decreases pericatheter localanesthetic leakage after continuous perineural infusions. Letter to the Editor. AnesthAnalg. 2011; 113: 206. PMid:21700699 http://dx.doi.org/10.1213/ANE.0b013e31821d38fb

[9] Harper GK, Stafford MA, Hill DA. Minimum volume of local anaesthetic required tosurround each of the constituent nerves of the axillary brachial plexus, using ultrasound guidance: A pilot study. Br J of Anaesth. 2010; 104: 633-636. PMid:20233750 http://dx.doi.org/10.1093/bja/aeq050

[10] Andersen HL, Andersen SL, Tranum-Jensen J. Injection inside the paraneural sheath of the sciatic nerve: Direct comparison among ultrasound imaging, macroscopicanatomy, and histologic analysis. Reg Anesth Pain Med. 2012; 37: 410-414. PMid:22609646 http://dx.doi.org/10.1097/AAP.0b013e31825145f3

[11] Schoenmakers KP, Wegener JT, Stienstra R. Effect of local anesthetic volume (15vs $40 \mathrm{~mL}$ ) on the duration of ultrasound-guided single shot axillary brachial plexusblock: A prospective randomized, observer-blinded Reg Anesth Pain Med. 2012 ; 37: $242-247$. PMid:22266898 http://dx.doi.org/10.1097/AAP.0b013e3182405df9

[12] Tran de QH, Dugani S, Pham K, Al-Shaafi A, Finlayson RJ. A randomized comparison between subepineural and conventional ultrasound-guided popliteal sciatic nerve block. RegAnesth Pain Med. 2011; 36: 548-552. PMid:22005661

http://dx.doi.org/10.1097/AAP.0b013e318235f566 